Relations industrielles

Industrial Relations

\title{
Colette Bernier avec la coll. de Catherine Teiger, Le travail en
} mutation

\section{Bernard Solasse}

Volume 46, numéro 3, 1991

URI : https://id.erudit.org/iderudit/050709ar

DOI : https://doi.org/10.7202/050709ar

Aller au sommaire du numéro

Éditeur(s)

Département des relations industrielles de l'Université Laval

ISSN

0034-379X (imprimé)

1703-8138 (numérique)

Découvrir la revue

Citer ce compte rendu

Solasse, B. (1991). Compte rendu de [Colette Bernier avec la coll. de Catherine Teiger, Le travail en mutation]. Relations industrielles / Industrial Relations, 46(3), 673-674. https://doi.org/10.7202/050709ar

Tous droits réservés @ Département des relations industrielles de l'Université Laval, 1991
Ce document est protégé par la loi sur le droit d'auteur. L'utilisation des services d'Érudit (y compris la reproduction) est assujettie à sa politique d'utilisation que vous pouvez consulter en ligne.

https://apropos.erudit.org/fr/usagers/politique-dutilisation/ 
problématique du vieillissement au travail peut apparaître différente. Même si l'approche scientifique et analytique, représentée par les nombreux chercheurs présents, domine largement, la perspective syndicale, centrée sur la solidarité, la défense des droits des travailleurs et le recours à l'État-providence est bien mise en valeur; par contre, celle des employeurs pour qui le vieillissement est également un enjeu important (croissance du coût salarial, adaptation à un environnement économique et technologique en rapide évolution, etc.) est moins prise en considération. Néanmoins, ce colloque de l'IRAT représente une contribution majeure à l'étude du vieillissement au travail et les actes, publiés sous la direction d'Hélène David, constituent une source de référence essentielle pour tout chercheur, enseignant, praticien ou syndicaliste concerné par ce sujet.

Gilles GUÉRIN

Université de Montréal

Le travail en mutation. Nouvelles technologies, qualifications et formation dans les emplois du secteur tertiaire au Québec, par Colette Bernier avec la collaboration de Catherine Teiger, Montréal, Éditions Saint-Martin, 1990, 168 p., ISBN 2-89035-171-8

Il s'agit en fait, de trois études «sectorielles» qui s'intitulent:

1) «Le secteur bancaire, polyvalence et spécialisation des emplois.»

2) «Le secteur des entreprises de service public. Formation en entreprise ou en établissement scolaire?»

3) «Le secteur de l'éducation = intégration des systèmes informatiques et spécialisation des tâches.»

À propos du secteur bancaire, l'auteur conclue au développement de la polyvalence des emplois notamment à partir de «l'étude de l'ancien poste de caissière» et d' «une spécialisation de certains postes dans les services d'épargne et de crédit» (p. 45).

L'étude se limite, il importe de le souligner, à certains postes de caissières et de préposés à l'épargne et au crédit. L'échantillon retenu et traité permet-il de conclure quand il s'agit du secteur bancaire à «la polyvalence et à la spécialisation des emplois»: encore que l'auteur nous dise avoir retenu "plusieurs niveaux d'analyse», soit «le secteur de l'entreprise et le poste de travail» (note infrapaginale p. 25).

De l'étude «des entreprises de service public» dont il est dit, sans plus de précision, qu'elles couvrent «quelques grands monopoles dont les services de téléphone, de gaz et d'électricité» (p. 55), ce qui fait beaucoup, se dégage non pas une conclusion mais une question: «Formation en entreprise ou en établissement scolaire?»

Et le lecteur d'apprendre à partir de la page 59 que l'auteur s'est surtout attardé à l'étude du poste «de commis à la facturation».

De l'étude du secteur de l'éducation l'auteur nous dit à la page 87 qu'il comporte trois niveaux distincts d'emplois: emplois de soutien administratif, emplois de soutien technique et emplois professionnels».

Et de préciser à la page 89 , nous citons et soulignons: 
«En fait, sur la base d'une dizaine d'études de postes, de différents niveaux et appartenant à différents services [lesquels?] des trois types d'institutions, nous pensons pouvoir conclure à l'existence de deux modèles. Le premier s'appliquerait aux personnels moins qualifiés des commissions scolaires où une certaine polyvalence serait recherchée de la part de ces personnels pour faire face à la complexification des emplois. Le second modèle serait celui retenu dans les cégeps, dans les universités où l'on tente de donner plus d'importance au groupe des techniciens et techniciennes, les croyant plus aptes à faire face aux nouvelles exigences des emplois.»

Une dizaine de postes étudiés qui relèvent de différents niveaux, de différents services et de trois types d'institutions, commissions scolaires, cégeps et universités. Voilà assurément un échantillon abondant!

Il faut cependant rendre justice à l'auteur ne serait-ce qu'en rapportant cette limite dont elle fait elle-même état, lorsqu'elle écrit à la page 142:

«Nous ne prétendons pas en arriver à des conclusions généralisables à l'ensemble du secteur tertiaire au Québec.»

Mais alors, pourquoi le sous-titre général de l'ouvrage et pourquoi les titres apportés à l'étude des trois (sous) facteurs considérés, des titres qui par ailleurs, sont eux-mêmes autant de conclusions?

Une dernière remarque pour terminer:

Le «tertiaire» de toute évidence ne saurait se limiter aux banques (et aux caisses populaires), aux services téléphoniques, au gaz, à l'électricité, au secteur de l'enseignement. Qu'en est-il du transport, des établissements commerciaux, des chaînes de distribution alimentaire, de l'hôtellerie, de la restauration? Des emplois «McDonald», des emplois, nous dit-on, précaires, déqualifiés, sous-payés, mais qui permettent de gonfler des statistiques de l'emploi, si chères à nos politiciens?

Bernard SOLASSE

Université Laval

La communication interpersonnelle et organisationnelle: l'effet Palo-Alto, par P. Dionne et G. Ouellet, Boucherville (Québec), Gaëtan Morin éditeur, 1990, 144 p.,

ISBN 2-89105-334-6

Neuf ans après La gestion des équipes de travail publié chez Gaëtan Morin, en passant par la soutenance de leurs thèses de doctorat et de quelques articles portant sur la négociation collective, Dionne et Ouellet présentent, cette fois en co-édition franco-québécoise (Gaëtan Morin et Éditions d'organisation), un ouvrage plus général où ils précisent et développent le cadre théorique qui sert d'assise à leur pratique professionnele en consultation organisationnelle.

Ceux et celles qui ont lu le premier ouvrage constateront que les auteurs, même s'ils se situent toujours dans la perspective de la «pragmatique de la communication», l'exploitent avec beaucoup plus d'envergure, de précision, et de maîtrise. Il y a aussi progression dans un style d'écriture original qui déjà leur était caractéristique: simple et léger mais aussi sérieux et réfléchi, insolent et quelquefois mordant mais aussi profondément sensible aux vicissitudes de la condition humaine, avec, en filigrane, un humour qui ne se dément qu'en de rares occasions. 